\title{
¿Existe el fraude en medicina?
}

\section{Samuel Karchmer K, ${ }^{1}$ Laura Romina Carpio Martínez ${ }^{2}$}

La medicina es un arte y a la vez una ciencia. Es la actividad humana más intensamente moral porque se fundamenta en una devoción plasmada desde hace 2,500 años en el juramento que todos los médicos hacemos al culminar la carrera de medicina, prometiendo una dedicación total a nuestros pacientes y servicio para el bien común de la sociedad. La medicina es, en esencia, una empresa moral fundamentada en un compromiso de confianza y honestidad. Este obliga al médico a mantener una elevada competencia profesional para utilizarla en beneficio del individuo. Los médicos, por lo tanto, están intelectual y moralmente obligados a estar al servicio del enfermo en todo momento y ocasión.

Hoy, tal compromiso se ve seriamente amenazado. La medicina mercantilizada trata a las personas como centro de lucro. Los médicos son empujados mediante amenazas y sobornos a abdicar su obligación con el paciente y a hacer a un lado a los más enfermos, cuyo cuidado puede no ser rentable. ${ }^{1}$

En el mundo actual de los negocios, los intereses económicos están superando a los valores éticos. El incremento tan importante en los costos de los servicios médicos está siendo un factor de incremento en actos de corrupción y fraude. En 2010, La Organización Mundial de la Salud citó al fraude como una de las diez principales causas de la ineficiencia en la atención sanitaria. El volumen de los gastos de atención médica en el sector salud a nivel mundial que se pierde a causa del fraude tanto en el sector privado

\footnotetext{
${ }^{1}$ Director médico. Director del Centro Especializado para la Atención de la Mujer. Profesor titular de la Especialidad en Ginecología y Obstetricia, UNAM.

2 Residente de cuarto año de la Especialidad en Ginecología y Obstetricia, UNAM.
}

Hospital Ángeles Lomas.

Correspondencia:

Samuel Karchmer K

Correo electrónico: s.karchmer@saludangeles.com

Aceptado: 27-09-2016.

Este artículo puede ser consultado en versión completa en http:// www.medigraphic.com/actamedica como institucional es, en promedio, de un $7.29 \%$ del gasto en recursos sanitarios, lo que equivale a 415 billones de dólares a nivel mundial.

Al médico, por el hecho de trabajar con seres humanos en beneficio de la salud, se le supone honesto y cabal en el más estricto sentido de las palabras. No se concibe a un médico fraudulento, y la idea de deshonestidad es repugnante -incluso, podemos decir que los médicos realizan un acto de fe al tomar como garantizada la integridad de sus colegas-.

La industria de la medicina la convierte hoy en día en un producto de mercado: de ahí deriva el uso excesivo de tecnologías médicas y medicamentos, y se ha sustituido a la medicina general por la especializada, la cual debería restringirse sólo para los casos en que esté justificada. ${ }^{2}$

\section{DEFINICIÓN}

El fraude en medicina es una de las faltas más graves que puede cometer un médico, sea clínico, investigador, docente o académico, y es inaceptable. El Diccionario de la Real Academia Española define al fraude como "la acción contraria a la verdad y a la rectitud, que perjudica a la persona contra quien se comete". En general, se entiende por fraude el engaño o la inexactitud de algo de manera consciente. Por otra parte, según la Academia Nacional de Ciencia de los Estados Unidos, se considera fraude la fabricación, falsificación y el plagio en la propuesta, ejecución o comunicación de un acto científico.

La Food and Drug Administration (FDA) de los Estados Unidos ha definido el fraude científico desde el año 2003 como "el anuncio, registro y/o reporte intencional de información o datos prefabricados falsos o delusorios dentro de las actividades pertinentes a la salud y/o al proyecto o estudio de la ciencia". También podemos hablar de fraude cuando se retiene información de un acto de negligencia médica.

Explicado en otras palabras, el fraude en medicina es una manipulación de la información médica con la finalidad de generar un beneficio al que lo comete. ${ }^{3}$

\section{EL FRAUDE DESDE UN ASPECTO ÉTICO}

El valor de la veracidad exige que en todo momento se diga la verdad, y lo que ofrece o trata de ofrecer la mentira 
es una impresión ajena a la verdad. La mentira puede ser inocente y hasta piadosa; el engaño lleva implícito el deseo de confundir al interlocutor, pero el fraude es claramente un delito encaminado a obtener un beneficio perjudicando al afectado. Aun así, estas tres categorías de falta a la verdad son éticamente reprobables.

Se puede decir que de todas las violaciones éticas, el fraude es, posiblemente, la más abominable, ya que es una alteración deliberada y voluntaria de la verdad, tanto en su sentido intelectual como moral.

El daño causado por el fraude en medicina — que cada vez se realiza con más frecuencia- constituye en nuestros días un impacto social importante, ya que gracias a él existe una depreciación de la confianza ciega que se tenía en el pasado al médico, no sólo dentro de la comunidad científica, sino también en el seno del público. Ya no existe la confianza en que los médicos siempre van a buscar la verdad y decirla si tienen la suerte de encontrarla. La verdad es la moneda del reino de medicina, de modo que quien comete un fraude en medicina equivale al falsificador de moneda, al fabricante de autos con graves defectos que los conoce pero los oculta, al que vende yerbitas para tratar tumores cancerosos y al político que adultera los resultados de un sufragio, ya que estos cinco perjudican a todos como sociedad. ${ }^{4}$

Sin embargo, un fraude en medicina no es un delito que pueda cometer cualquiera: es una estafa perpetrada con pericia. Para cometerla es necesario saber lo suficiente para engañar a varios sin que se den cuenta. Quizás, el fraude en medicina ocurre con mayor frecuencia que en otras ciencias de la salud debido a que los médicos no son entrenados como "científicos" sino como "artesanos", de modo que se engañan y autoengañan más fácilmente que en otras áreas científicas. ${ }^{5}$

\section{EL FRAUDE EN PUBLICACIONES MÉDICAS}

Los médicos son sometidos a una gran presión para publicar desde que son estudiantes, mucho más frecuentemente que en cualquier otra área científica. En los orígenes de la ciencia, la única motivación en la búsqueda científica era la simple curiosidad, el deseo de saber más sobre algo concreto. Hoy, la relación entre ciencia y sociedad ha cambiado.

Las investigaciones actuales requieren medios materiales y personales que son muy costosos desde el punto de vista económico, lo que obliga a que dependan de la financiación de la administración pública o empresas privadas con intereses muy particulares. En este contexto, los científicos están obligados a competir por los recursos necesarios para llevar a cabo las investigaciones y publicar los resultados lo antes posible, con vistas a un mayor reconocimiento científico en la materia estudiada; sólo así se pueden ase- gurar nuevas fuentes de financiación para investigaciones futuras. Esta presión por publicar ha llevado a que algunos autores caigan en el error de rebajar la calidad ética de sus trabajos, llegando en algunos casos al fraude científico. En este sentido, debemos recordar que el proceso de publicación de un artículo se basa en la credibilidad, verdad, autenticidad y honestidad científicas.

En cuanto al fraude en las publicaciones médicas científicas, no hay un consenso en cuanto a definición y clasificación del mismo; por este motivo, se le suele agrupar junto con las fallas graves, malas prácticas menores y otros aspectos de la desviación ética de la actividad médica bajo el epígrafe de "mala praxis médica". ${ }^{6}$

El fraude en las publicaciones médicas se puede realizar mediante:

\section{Manipulación de los datos reportados en la realización y/o los resultados de un estudio}

Cuando se realiza un estudio pero sólo se publica parte de la totalidad de los datos recabados, cuando se reportan observaciones inventadas no basadas en los métodos presentados en el informe de la investigación o cuando se presentan resultados totalmente imaginarios basados en observaciones ficticias se está realizando un fraude.

Se puede llevar a cabo de manera fraudulenta la modificación de los datos u observaciones recabados en una investigación donde los datos correctos existen, pero los autores cambian los valores con el fin de obtener resultados que corroboren sus hipótesis. Para lograr esto, se tiende a exagerar ciertos resultados y se ocultan malévolamente otros.

La técnica del "meat extender" es un fraude común que consiste en agregar uno o más datos a una serie de casos ya publicada, sin declarar que la mayoría de ellos ya han sido descritos en comunicaciones previas; es decir, sin citar dichos textos, haciendo creer que toda la información o los casos fueron realizados por el autor, cuando lo único que hizo fue extender dicho estudio o serie de casos. ${ }^{7}$

Una falta comúnmente cometida, que también se toma como fraude al momento de publicar, es no buscar de manera extensa en la literatura global el tema a tratar en el estudio y desconocer los predecesores intelectuales de algún tema o publicación, dejando fuera referencias que indican indagaciones previas u omitiendo citas relevantes.

Otro defecto común en los trabajos de investigación es publicar de manera duplicada, parcial o totalmente, bien sea en documento impreso o electrónico, sin informar a todos los editores que han participado en dicho estudio.

También, fraudulentamente, se pueden dar a conocer los resultados de una investigación antes de ser revisada 
o publicada; por lo general, esto se hace de una forma sensacionalista, para ganar prestigio. ${ }^{8}$

$$
\text { Plagio }
$$

La norma ética considera autor o coautor de un trabajo a quien ha contribuido sustancial y cabalmente en alguna o todas las fases para lograr su finalización exitosa. Estas fases incluyen desde la generación de la idea hasta su publicación y divulgación por cualquier medio.

El plagio es la presentación total de un proyecto de investigación, un borrador, un artículo u otro texto creado por alguien más como si hubiera sido original de quien lo publicó. El plagio es menos realizado hoy en día porque es fácil detectarlo; sin embargo, aún existe. Se puede decir que el plagio es la apropiación de ideas o textos para darlos a conocer como propios sin obtener permiso, sin citar ni dar los debidos créditos a los autores.

Los plagiarios de publicaciones médicas no son más que meros rateros que difunden información científica de manera casi tan buena como los autores originales. Generalmente, estos médicos roban información, pero apenas la adulteran, de modo que su delito no se propaga ni perjudica más que a los autores originales. ${ }^{7}$

\section{Autoría ficticia}

La autoría ficticia se presenta cuando se incluye a una o más personas que no han colaborado con un trabajo de investigación.7

Existe la autoría regalada, que ocurre muy comúnmente en publicaciones científicas y se hace para pagar algún favor, halagar a algún superior (jefe) o, en algunos casos, por mandato expreso del mismo. Hay algunos casos de autoría regalada donde el autor quiere incluir a algún familiar o amigo para aumentarle (falsamente) su currículo o, peor aún, simplemente para darle inmerecida fama.

La autoría honoraria es otro tipo de autoría ficticia, que se proporciona a personas que, en razón de su importancia y fama (científica, política o social), se considera que deben aparecer como autoras del trabajo para darle mayor peso a la publicación.

Por último, la autoría canjeada o intercambio recíproco de autorías consiste en: "yo te incluyo como autor en un artículo mío si tú me incluyes como autor en un artículo tuyo". Esto puede ocurrir entre colegas, aunque también puede pasar entre instituciones y países diferentes. ${ }^{9}$

Toda autoría ficticia debe ser evitada a toda costa, ya que además de ser una falta ética, se corre el riesgo de que las publicaciones o trabajos con autorías ficticias sean luego declarados como fraude científico y sean éticamente penalizados, socialmente estigmatizados y/o legalmente sancionados. Lo más grave es que, en muchos casos, los autores ficticios ni siquiera saben en cuáles publicaciones están apareciendo, pero al presentarse como autores de un trabajo, asumen la responsabilidad de su contenido.

Hay muchos casos de famosos médicos que han quedado envueltos en casos de fraude científico sin realmente haber participado en él, por el solo hecho de haber aceptado ser coautores de trabajos en los cuales no habían intervenido. $^{8}$

En el año 2005, se realizó una investigación mediante una encuesta aplicada a 3,600 médicos en la mitad de su carrera y a 4,160 médicos que ejercían su profesión. Dicha encuesta indagaba acerca de los fraudes en las publicaciones y la obtención de la información para sus trabajos de investigación. Se observó una menor tasa de "fraude" en los estudiantes, y la causa se debió principalmente al temor a ser descubiertos o sancionados. En el grupo de los médicos que ejercían la medicina, el 38\% reconoció que había cometido mala conducta en sus publicaciones o trabajos de investigación, en contraste con el grupo de estudiantes en la mitad de su carrera, donde sólo el $28 \%$ admitió haber incurrido en ella. De las respuestas que se obtuvieron en la encuesta, se concluyó que los tipos de mala conducta más frecuentes en los trabajos de investigación y las publicaciones fueron:

1. Falsificación de datos de investigación.

2. Uso de las ideas de otros sin obtener permiso o dar los debidos créditos.

3. Empleo no autorizado de la información de una investigación.

4. Cambio del diseño, metodología o resultados de un estudio en respuesta a presiones de la fuente de financiamiento.

5. Publicación de los mismos datos o resultados en dos o más comunicados.

6. Asignación inapropiada de autoría.

7. Utilización de diseños de estudio inadecuados.

De todos los participantes, 33\% dijeron que habían realizado al menos dos de estas malas conductas durante los tres años anteriores.

Se puede decir que el fraude en las publicaciones científicas en el ámbito médico ha sido objeto de numerosos artículos, informes, denuncias y extensos ensayos y libros. Este hecho, aparentemente insólito, ha despertado el interés de científicos, universidades, institutos, psicólogos, sociólogos y filósofos. ${ }^{3}$

Sorprende que en nuestro país, este tema no haya sido estudiado por instituciones como el Consejo Nacional de Ciencia y Tecnología (CONACYT) o la Academia Mexicana de Ciencias (AMC), y que a los pocos casos que han salido 
a la luz pública no se les ha concedido la importancia que merecen. En las naciones científicamente desarrolladas, ya se han establecido oficinas y comités encargados de analizar y sancionar las conductas impropias de los médicos investigadores, mientras que en otros, como el nuestro, este tema no ha despertado la atención que merece. ${ }^{10}$

Un artículo reciente publicado en la revista Science informa que durante 2004 y 2005 se descubrieron 60 científicos que incurrieron en un quehacer inapropiado. Las acciones fraudulentas más comunes fueron la falsificación de los resultados $(40 \%)$, plagio $(34 \%)$, fabricación o robo de datos $(7 \%)$ y otros (19\%). Esto demuestra que el fenómeno de fraude en las publicaciones científicas tiene lugar en cualquier país del mundo donde se realice ciencia. ${ }^{11}$

\section{HISTORIAS DE FRAUDE EN LA INVESTIGACIÓN BIOMÉDICA}

A continuación se expone un relato breve de algunos de los casos de fraude científico más llamativos que han salido a la luz pública en los últimos 20 años.

\section{Ranjit Kumar Chandra}

Chandra era un experto de renombre universal en el campo de la nutrición y la inmunología que fue formalmente acusado de haber cometido fraude científico por el British Medical Journal. El investigador había publicado un artículo en la revista Nutrition en el año 2001 donde afirmaba que una fórmula multivitamínica que había patentado podía revertir los problemas de memoria en personas mayores de 65 años.

Sin embargo, el mismo artículo había sido enviado previamente para su consideración al British Medical Journal en el año 2000 y había sido rechazado después de una revisión por expertos estadísticos que afirmaron que dicho estudio reunía las características necesarias para hacer pensar que había sido inventado completamente.

El British Medical Journal solicitó a la Memorial University of Newfoundland que investigara el caso. El panel universitario determinó que el trabajo no podía haber sido realizado como Chandra había descrito, y cuando se le pidió al investigador que mostrara sus datos originales al comité, él afirmó que la universidad los había extraviado. En el verano del año 2002, Chandra acabó abandonando la Memorial University of Newfoundland de Canadá bajo la sospecha de fraude. En 2005, el estudio de Chandra de las vitaminas fue formalmente rechazado y se inició una investigación de sus artículos anteriores.

La historia lamentable de la carrera científica de Chandra se remonta a finales de 1980, cuando fue contratado para realizar un trabajo para la compañía americana Ross
Pharmaceuticals. Esta compañía deseaba averiguar si una fórmula nutricional que había diseñado podía ayudar a los neonatos a evitar la aparición de alergias y decidió encargar el estudio a Chandra. Se identificaron 288 neonatos cuyos padres tenían una historia clínica de alergias y desearon participar en la investigación.

Paralelamente, las compañías de alimentación Nestlé y Mead Johnson también se pusieron en contacto con Chandra para hacer análisis similares a partir de sus fórmulas infantiles. Un año después, a pesar de no haber reunido los datos necesarios para el informe, Chandra publicó el trabajo de Nestlé y, posteriormente, los otros dos. Aunque teóricamente había utilizado ingredientes casi idénticos, Chandra reportó que las fórmulas de Nestlé y Mead Johnson podían prevenir la aparición de alergia en los neonatos, mientras que la de Ross Pharmaceuticals no. Cuando se le exigió que explicara tal discrepancia, el investigador aseveró que el estudio de Ross no había sido diseñado apropiadamente y que, además, él no había recibido la remuneración suficiente para llevarlo a cabo de forma adecuada.

Después, el científico fue acusado de no haber realizado nunca los estudios que había publicado en varias revistas respetables, entre ellas el British Medical Journal.

Con la finalidad de presentar argumentos en su defensa, Chandra decidió difundir en la revista Nutrition Research, de la que él mismo era editor, un trabajo que confirmaba los resultados publicados previamente. Amrit Jain, el autor, laboraba en el Medical Clinic and Nursing Home en Jaipur, India, aunque después se constató que el lugar era ficticio. Por otra parte, cuando los investigadores intentaron ponerse en contacto con Jain, no obtuvieron respuesta alguna ni pudieron confirmar su existencia ni credenciales. ${ }^{12}$

\section{Eric Poehlman}

Poehlman es un investigador experto en el campo de la obesidad y el envejecimiento, y ha sido el primer científico de la historia de Estados Unidos encarcelado por falsificar datos en una solicitud de beca.

Empezó a trabajar en la Facultad de Medicina de la Universidad de Vermont en 1987 como profesor asociado y, posteriormente, laboró durante tres años en la Universidad de Maryland en Baltimore. El científico era considerado una autoridad mundial en el campo de los cambios metabólicos que aparecen como consecuencia del envejecimiento y la menopausia. En este sentido, fue autor de más de 200 artículos publicados durante dos décadas, dedicados fundamentalmente a la investigación sobre la genética de la obesidad y el impacto del ejercicio. Estos estudios incluían, a menudo, un seguimiento de los sujetos con la finalidad de documentar cambios en su fisiología. 
Su carrera meteórica se derrumbó cuando Walter De Nino, técnico de laboratorio al que Poehlman había tutelado, decidió denunciar que este había cometido fraude en varias de sus investigaciones. El científico fue acusado de mala conducta científica y, en marzo de 2005, se declaró culpable de los cargos que se le imputaban, reconociendo que había falsificado 17 solicitudes de beca financiadas por los National Institutes of Health y había manipulado e inventado resultados en al menos diez de los trabajos que había publicado en revistas científicas entre 1992 y el año 2000.

En 2006, Poehlman fue condenado a un año y un día de cárcel por el delito de utilización de datos falsos en becas de investigación. Además de la condena a prisión, el científico no podría solicitar más becas federales durante el resto de su vida y fue obligado a enviar cartas de retracción y corrección a varias revistas científicas en las que había publicado sus trabajos. ${ }^{12}$

\section{Jon Sudbo}

A principios del año 2006, la comunidad de científicos dedicada a la investigación del cáncer oral se quedó atónita tras conocer que el oncólogo Sudbo había falsificado datos en un artículo publicado y después retractado por The Lancet. Asimismo, luego de que aparecieran las primeras sospechas sobre este texto, el New England Journal of Medicine expresó también dudas sobre dos trabajos del mismo autor publicados en esta revista y, finalmente, fueron también retractados. Una investigación independiente sobre la totalidad de la producción científica de Sudbo desde su tesis de 1993 concluyó que la mayor parte de sus publicaciones eran inválidas debido a la fabricación y manipulación de datos.

Jon Sudbo era dentista y oncólogo del Hospital Radium de Oslo y, hasta febrero de 2006, había sido profesor asociado de la Universidad de Oslo. Después de que se conociera el escándalo mencionado, se le retiró la licencia para practicar medicina y odontología, y en el año 2006 se le retiró el título de doctor.

Los hechos que motivaron el descubrimiento del fraude de Sudbo se remontan a octubre de 2005, cuando se pudo constatar que el artículo enviado a revisión a The Lancet que concluía que los antiinflamatorios no esteroideos como el ibuprofeno disminuían el riesgo de cáncer oral en los fumadores se basaba en datos fraudulentos de los pacientes.

Con posterioridad, Sudbo admitió que había utilizado datos totalmente ficticios en al menos dos artículos publicados en el New England Journal of Medicine y el Journal of Clinical Oncology. Asimismo, un comité independiente de investigación liderado por el profesor sueco Anders Ekbom y que incluyó a un miembro del US National
Cancer Institute (algunos de los trabajos de Sudbo habían sido firmados por coautores estadounidenses y financiados con becas de tal organismo) fue el encargado de revisar el trabajo de Sudbo; en concreto, 38 artículos con 65 autores de seis países. De los 38 textos publicados desde 1993, 15 fueron considerados fraudulentos, incluyendo los correspondientes a su tesis doctoral. El comité no encontró ninguna evidencia de que los coautores de los trabajos estuvieran implicados en el fraude masivo. ${ }^{12}$

$$
\text { Hwang Woo Suk }
$$

Otro caso mundialmente famoso de fraude científico por manipulación de datos fue el del profesor Hwang Woo Suk, un científico surcoreano que es considerado por muchas personas el líder en la investigación en el campo de las células madre, a pesar de su falsificación sobre la clonación de embriones humanos.

Este individuo ganó fama mundial en el año 2005, cuando él y su equipo anunciaron que habían clonado embriones humanos y extraído células madre de los mismos. Poco tiempo después de que su trabajo fuera publicado en revistas de gran prestigio como Science y Stem cells, se pudo demostrar de manera concluyente que si bien Hwang había extraído células madre de embriones humanos, estos eran embriones normales y no clonados, lo que significaba que el procedimiento técnico de extracción de células madre era mucho más sencillo y ya se había conseguido previamente por otros investigadores.

Hwang y su grupo se habían convertido en el primer equipo en el mundo que clonaba un embrión humano con el fin de avanzar en la investigación médica científica. En su artículo publicado en mayo de 2005 en la revista "Science", Hwang confirmó haber creado una matriz de 11 células madre con el objetivo de desarrollar terapias para enfermedades crónicas. Con ello ilusionó a millones de personas que vieron más cercanas las posibilidades de cura de muchas enfermedades degenerativas como la diabetes o el Parkinson. Sin embargo, en diciembre de 2005, uno de sus colaboradores denunció que nueve de esas líneas celulares que había publicado eran falsas, algo que ha confirmado un informe publicado por expertos de la Universidad Nacional de Seúl. En realidad, en lugar de desarrollar células clonadas partiendo de las originales de los sujetos de prueba, las produjo a partir de un grupo de células guardadas in vitro.

Hwang aceptó la responsabilidad de sus falsas investigaciones, e incluso emitió una disculpa pública y renunció a su puesto como profesor en la Universidad Nacional de Seúl tras haber admitido que había tergiversado los resultados de al menos nueve de las 11 líneas de células madres que afirmó haber creado. 
Actualmente, Hwang está condenado a cárcel por el tribunal de Seúl, ya que se le acusó de malversación de fondos estatales y violación de leyes bioéticas. ${ }^{12}$

\section{William Griffith McBride}

Griffith McBride fue uno de los investigadores más famosos del mundo; logró su fama por alertar al mundo acerca de los efectos de la talidomida sobre los fetos. Sin embargo, fue acusado de falsificar informes sobre experimentos que afirmaban que el bendectin (Debendox) también podría dañar a los fetos.

Los cargos en contra de Griffith fueron presentados por Norman Swan, su colega de investigaciones. El juez que encabezó la pesquisa del caso declaró que McBride carecía de integridad profesional por la falsificación deliberada y la publicación de resultados adulterados en $1988 .{ }^{12}$

\section{EL FRAUDE EN MEDICINA CON FINES MERCADOTÉCNICOS}

En los últimos años, se ha venido ventilando la existencia de manipulación de los resultados o de la planeación y ejecución de investigaciones médicas con fines promocionales de algún medicamento. Es paradójico que la industria farmacéutica patrocine investigaciones para "crear" evidencia y obligar al uso de determinado fármaco fabricado por la misma, de acuerdo con las reglas de la medicina basada en evidencias. Es cierto que existen trabajos legítimos creados de esta forma, pero cada vez se documentan más casos de fraude. En virtud del compromiso generalizado de la profesión médica con la medicina basada en evidencias, estas conductas son particularmente graves y ponen en entredicho el compromiso básico con el paciente de indicar siempre el mejor tratamiento a su disposición.

Los casos documentados hasta la fecha son muchos, como el del rocecoxib, en que Merck ocultó la evidencia de su toxicidad y los autores firmaron artículos sin participar. ${ }^{10}$ Otro fue el escándalo de la reboxetina, un inhibidor de la receptación de norepinefrina utilizado para el tratamiento de la depresión que fue aprobado en muchos países europeos desde finales de los años 90. En 2010, un grupo de investigadores alemanes publicó en el British Medical Journal un estudio que demostraba que no sólo el medicamento era inefectivo, sino que, además, la compañía farmacéutica había ocultado a la comunidad médica aquellas pruebas que le eran desfavorables. De siete ensayos clínicos contra placebo, seis mostraban que la reboxetina no era más eficaz que el placebo. Ninguno de esos estudios fue publicado en revistas científicas. Además, en los ensayos clínicos contra otros antidepresivos, la compañía farmacéutica había eliminado tres cuartas partes de los datos. Inexplicablemente, en Europa la reboxetina sigue siendo comercializada por Pfizer bajo los nombres "Norebox" e "Irenor", aunque la FDA nunca autorizó la reboxetina por falta de eficacia probada.

Pfizer no es la única compañía envuelta en un escándalo de ocultación de datos científicos. En febrero de 2010, el senado de los EUA publicó un informe donde se describe cómo la farmacéutica GlaxoSmithKline (GSK) no sólo mintió sobre los riesgos cardiovasculares de uno de sus medicamentos contra la diabetes, la rosiglitazona, sino que además trató de silenciar a los científicos que los advirtieron. La FDA calcula que este fármaco produjo 83,000 infartos entre los años 1999 y 2007. Hace unos meses, GSK decidió declararse culpable ante los tribunales norteamericanos. El Dr. John Buse, profesor en la Universidad de Carolina del Norte, comenzó a advertir sobre los posibles riesgos de la rosiglitazona, un medicamento que suponía miles de millones de ingresos para GSK. ${ }^{13}$

La regulación de la industria farmacéutica es todavía deficiente. Por ejemplo, las compañías farmacéuticas no están obligadas a publicar todos los ensayos clínicos que realizan y, por lo tanto, los que se hacen públicos son casi siempre favorables a sus intereses. Es también una práctica habitual que se les haga firmar un contrato a los investigadores universitarios que aceptan financiación de una farmacéutica; por medio del mismo, se comprometen a no publicar ningún resultado sin el permiso de la compañía. Las farmacéuticas financian, además, numerosas revistas y conferencias médicas.

La solución no es sencilla, ya que se necesitarían investigaciones costosas sin conflicto de interés para verificar las bondades e indicación de un medicamento antes de ponerlo a la venta. Las revistas médicas deben verificar que no avalan o publican un comercial o un fraude. ${ }^{14}$

\section{MOTIVACIONES DEL MÉDICO PARA REALIZAR FRAUDE EN EL EJERCICIO DE SU PROFESIÓN}

Existen, principalmente, dos tipos de motivaciones que impulsan al médico para proceder en forma deshonesta en el ejercicio de la medicina. El primero es de tipo personal, con deseos de grandeza y admiración de los demás. El segundo grupo de factores que podrían impulsar al médico para violar los principios éticos del quehacer científico son las presiones impuestas por el entorno socioeconómico en el que se desarrolla la ciencia en la actualidad.

Para obtener reconocimiento científico, grados académicos, becas, remuneración monetaria o premios es necesario generar fama social en el ámbito médico. Para lograrla, hace falta un intenso trabajo de investigación, estudio constante y publicaciones científicas. Esta labor no es sencilla, requiere de mucho tiempo, esfuerzo y calidad. 
Con el propósito de acelerar y evadir la multitud de obstáculos que un médico debe enfrentar, algunos recurren a la falsificación, la mentira o el fraude.

A los médicos, el tener muchos pacientes, realizar mayores intervenciones médicas y quirúrgicas, así como publicar numerosos trabajos en revistas de gran prestigio les confieren puntos curriculares y reconocimiento en el ámbito médico y social. Por ello, algunos deciden recurrir al engaño para tener un mayor número de consultas, cirugías y/o trabajos publicados y obtener los beneficios que de eso se derivan. ${ }^{11}$

\section{LOS SEGUROS DE GASTOS MÉDICOS}

En las grandes corporaciones de aseguradoras de gastos médicos, donde se manejan miles de millones de dólares, se dan los fraudes cada vez con mayor frecuencia.

En Estados Unidos, las 1,300 compañías de seguros médicos representan un negocio de 300,000 millones de dólares anuales, y son ellas mismas las que niegan el aseguramiento a viejos o enfermos y, con frecuencia, se rehúsan a dar la atención médica a la que sus asegurados tendrían derecho. En México, cada año hay aproximadamente 250,000 reclamaciones a las aseguradoras por incumplimiento, y son el mejor ejemplo de los recursos desviados hacia excesos administrativos, recursos que deberían servir a los asegurados. En los últimos cinco años, el costo de los fraudes, sólo en seguros de gastos médicos, se estima en aproximadamente $\$ 9.5$ mil millones de pesos. ${ }^{15}$

Los seguros médicos privados tienen numerosas y diversas limitaciones: para ingresar es fácil si se es joven y sano, muy difícil y costoso si se pertenece a la tercera edad y si la aseguradora, al hacer el examen médico al candidato a asegurarse, encuentra algún dato que le haga sospechar de una enfermedad persistente; en esos casos, será raro que acepten asegurarlo, con lo que lo excluyen de la atención no sólo de su supuesta enfermedad, sino también de aquellas que a largo plazo pudieran ser consecuencia de la misma.

Las aseguradoras, con frecuencia, encuentran explicaciones o motivos para rehuir a sus obligaciones; dentro de ellos destacan desde padecimientos congénitos, la obligación de dejar transcurrir un periodo de espera para ser amparados o enfermedades preexistentes hasta causas como la falta de desglose o el estado de cuenta de facturas, gastos que no exceden el deducible contratado o beneficios que rebasan lo contratado por la póliza (terapia física, prótesis, consultas intrahospitalarias e, incluso, maternidad).

En ocasiones, después de que un paciente se ha realizado estudios a causa de una supuesta enfermedad o accidente, si los resultados son negativos, la aseguradora se niega a pagar el costo de los exámenes, aduciendo que fueron hechos como un "chequeo" de salud. ${ }^{16}$
Una de las limitaciones más graves que sufre un asegurado es que debe renovar su póliza de seguro en forma anual; si unos meses antes de la renovación la persona sufre un padecimiento grave, la aseguradora acepta la siguiente renovación, pero excluyendo esa patología o las enfermedades que pudieran derivar de la misma; o, en todo caso, la renovación supone una prima mucho más elevada que la anterior. ${ }^{17}$

Una variedad — hoy en día muy común- de los seguros privados es aquella en la que el individuo acepta ser atendido por un grupo de médicos que son empleados de la aseguradora. Aquí las limitaciones son las instrucciones que se dan a estos médicos empleados: deben restringir el uso de medicamentos costosos, hacer el mínimo de exámenes de laboratorio y gabinete, y evitar el internamiento en hospitales. En este último caso, el aseguramiento no sólo es limitado, sino que se ocasiona un conflicto de intereses, ya que la aseguradora no vela por el bienestar del sujeto y la conservación de su salud, sino por sus intereses comerciales. Esta figura es conocida como médico "gate keeper"; es decir, la función del portero en un equipo de fútbol: parar los goles.

En el año 2004 se realizó una encuesta cuyo resultado fue publicado en la Gaceta Médica de México, relativo a la experiencia de médicos en tres ciudades de la república y su relación con compañías de seguros en México. En la encuesta fue claro no sólo que el estipendio por sus servicios representaba una mínima parte de sus honorarios habituales, sino también que existían dificultades para aceptar la realización del servicio médico y tardanza en los pagos. ${ }^{17}$

\section{FRAUDE EN LOS MEDICAMENTOS}

Uno de cada diez medicamentos que se comercializan es falso, según datos de la Organización Mundial de la Salud (OMS). Esta actividad ilegal, de consecuencias letales para la población de todo el mundo, mueve cada año entre 30,000 y 40,000 millones de dólares.

El mercado de los medicamentos falsificados constituye un peligro real y presente para la salud pública. Los falsificadores actúan de modo ilegal y no tienen interés alguno en la seguridad de los pacientes. Se infiltran en la cadena de suministro de los medicamentos legítimos y también utilizan farmacias por internet, sin autorización, para ocultar sus fármacos falsos entre los medicamentos legales.

El consumo de fármacos falsificados o de calidad inferior puede suponer un riesgo para la salud. Una persona que tome de forma habitual medicamentos falsificados puede no estar aprovechando los efectos terapéuticos del fármaco, y existe la posibilidad de que aparezcan resistencias farmacológicas o de que se produzca un problema más grave. Los medicamentos falsificados son un problema de salud: 
su utilización puede ocasionar daños a la población; en algunos casos, incluso la muerte. Estos medicamentos presentan una falta de eficacia, carecen total o parcialmente del principio activo y/o pueden generar un efecto tóxico.

Se consideran medicamentos irregulares los fármacos caducos, robados, adulterados, contaminados o falsificados. Un medicamento irregular incumple con la Ley General de Salud, el Reglamento de Insumos para la Salud y la NOM-059-SSA1-2013, de buenas prácticas de fabricación de medicamentos. Se puede sospechar de un fármaco presuntamente falsificado si nos encontramos frente a un empaque sin número de lote, fecha de caducidad o registro sanitario. Es importante tener presente que estos datos siempre son indelebles y nunca están colocados con etiquetas autoadheribles.

México ocupa el sexto lugar a nivel mundial en fabricación y consumo de medicamentos falsificados o de calidad inferior, por lo que en los últimos tiempos ha aumentado de forma notable el interés por el riesgo que suponen. Por ejemplo, en febrero de 2015, la Comisión de Salud del Senado aprobó, con modificaciones, una minuta de la Cámara de Diputados que modifica la Ley General de Salud, para sancionar hasta con nueve años de prisión a quienes vendan, comercien o distribuyan fármacos caducos.

También se castiga con uno y hasta nueve años de cárcel y multa de 20 mil a 50 mil días de salario mínimo a quien falsifique, adultere o permita la adulteración de material para envase, etiquetamiento o empaque de medicamentos. Asimismo, se aplican de uno a nueve años de prisión a quienes vendan, comercien, distribuyan o transporten medicamentos falsificados, contaminados o con caducidad vencida.

Podemos concluir que la profesión médica debe reafirmar la primacía de su obligación con el paciente, ya que hoy en día sus crecientes intereses económicos oscurecen nuestra obligación y amenazan con transformar la atención de la salud, cambiándola de un compromiso de confianza a un contrato de negocios que pisotea los valores éticos y morales.

El fraude en medicina es la deformación de la verdad en todo lo que atañe a la función médica con el objetivo de obtener alguna ganancia, ya sea monetaria, de prestigio o de protección. La mejor manera de evitar el fraude en medicina es a través de la educación, la capacitación y la formación del personal médico, especialmente en el ámbito de la conducta ética y la investigación.

\section{REFERENCIAS}

1. Schulz PC, Katime K. Los fraudes científicos. Revista Iberoamericana de Polímeros. 2003; 4 (2): 1-90.

2. National Health Accounts [online database]. 2010 [Consultado el 7 de julio]. Geneva, World Health Organization. Disponible en: http:// www.who.int/nha.

3. Salinas P. Fraude científico en el ambiente universitario. Rev Fac Med Universidad de Los Andes. 2005; 13: 42.

4. Geyman J. The Corrosion of Medicine: Can the Profession Reclaim its Moral Legacy? Common Courage Press; 2008.

5. Pérez J. Publicaciones científicas: ¿aspectos éticos o fraude? Nutr Hosp. 2009; 24 (4): 512.

6. Alfonso F, Bermejo J, Segovia J. Cambiando el énfasis: de la uniformidad de los requisitos técnicos a los aspectos bioéticos. Rev Esp Cardiol. 2004; 57: 592-593.

7. Hernández C. Ética en la publicación científica. Rev Haban Cienc Med. 2007; 6 (5): 10.

8. Avanzas P, Bayes GA, Pérez IL, Sanchis J, Heras M. Consideraciones éticas de la publicación de artículos científicos. Rev Esp Cardiol. 2011; 64 (5): 427-429.

9. Hernández CF. Fraude en la autoría de artículos científicos. Rev Biomed. 2007; 18: 127-140.

10. Laredo F. La manipulación de la evidencia científica en la medicina con fines mercadotécnicos. Med Int Mex. 2011; 27 (5): 419.

11. Benítez BL. La mentira, el engaño y el fraude en la ciencia. Acta Médica Grupo Ángeles. 2005; (3): 3.

12. Bosch X. Historia reciente del fraude en la investigación biomédica. Sociedad Española de Bioquímica y Biología Molecular. 2008; 156 (1): 34.

13. Angell M. The truth about the drug companies. New York: Random House; 2004.

14. Pinto E. A propósito de casos de fraude en la industria farmacéutica. Rev Peru Med Exp Salud Pública. 2013; 30 (1): 146.

15. Aguilera M, Calderón M, González VM, Rosas RN, Lozano LG, Aguilar $\mathrm{BP}$ et al. Comisión Nacional de Seguros y Finanzas. Anuario Estadístico de Seguros y Fianzas; 2010.

16. Rivero O, Martínez LA. La medicina actual. Los grandes avances y los cambios de paradigma. Rev Fac Med UNAM. 2011; 54 (2): 21.

17. Rivero S, Tanimoto M, Paredes R. Los médicos especialistas y el problema de honorarios y seguros médicos. Gac Med Mex. 2003; 139 (3): 291. 